\title{
Efecto de los factores ambientales sobre la diversidad de insectos hemimetábolos y coleópteros acuáticos en la cuenca del Río Xichú, Guanajuato, México
}

\author{
Ulises Torres-García ${ }^{1}$, Cloe X. Pérez-Valladares ${ }^{2 *}$, Yvonne Herrería-Diego ${ }^{2}$ \& \\ Raúl F. Pineda-López ${ }^{1}$ \\ 1. Facultad de Ciencias Naturales, Universidad Autónoma de Querétaro, Querétaro, México; ulisestoga@gmail.com, \\ rufuspinedal@gmail.com \\ 2. Facultad de Biología, Universidad Michoacana de San Nicolás de Hidalgo, Morelia, México; \\ cloe.persephone@gmail.com* \\ * Correspondence
}

Recibido 12-XII-2013. Corregido 20-I-2014. Aceptado 13-II-2014.

\begin{abstract}
Effects of environmental factors over aquatic hemimetabola and Coleoptera diversity in the Xichu's basin, Guanajuato, México. Lotic systems, in particular temporal streams, represent an important proportion of continental waters, but are poorly studied. This is particularly clear in central Mexico, where temporal streams are abundant and remain poor studied despite their great potential for high diversity and the important energy within these ecosystems. The aim of this study was to analyze the environmental variables and scales at which these variables have effects on aquatic insect assemblages in a semiarid fluvial system in Central Mexico. A total of 89 genera were registered, the order Coleoptera showing the highest richness. Peak values for alpha diversity were found on permanent sites in the dry season $\left({ }^{1} \mathrm{D}=10.63\right)$, the more stable environment in permanent streams during the dry season might accounted for this pattern. Assemblages at our study sites showed low similarity $\left(\mathrm{I}_{\mathrm{j}}<0.45\right)$ indicating high genera replacement among sites, possibly related to differences in hydroperiod. Partition analysis indicates that variation in genera composition among rivers contributed a large proportion of the diversity (37\%). Results from the canonical correspondence analysis point to mesohabitat, velocity, ecological quality, and hydrological order, as the most important variables explaining diversity patterns. In addition, hydrological condition and intermittency were important factors related to diversity $(\sim 21-22 \%)$. The results suggest that permanent sites function has species sources for intermittent sites, highlighting the need for conservation planning at the landscape level in the Xichu's basin. Rev. Biol. Trop. 62 (Suppl. 2): 69-80. Epub 2014 April 01.
\end{abstract}

Key words: diversity partition, beta diversity, hydroperiod, temporal streams, ecological scales.

A pesar de constituir ecosistemas acuáticos bastante comunes a nivel mundial, las redes fluviales integradas por cauces permanentes e intermitentes aún permanecen poco exploradas (Larned, Thibault, Arscott \& Tockner, 2010). Esta variación en la hidro-periodicidad o flujo ha sido señalada como una fuente de perturbación natural que altera la estructura ambiental y la distribución de las comunidades dulceacuícolas (Bonada, Rieradevall \& Prat, 2007), debido principalmente a una causa combinada de la preferencia del hábitat y de los cambios inducidos por los niveles cambiantes del agua en el tiempo y el espacio (Fairchild, Cruz \& Faulds, 2003; Klečka, 2008). También influyen las afectaciones antrópicas (Feld \& Hering, 2007), el tipo de sustrato (Acuña, Muñoz, Giordi, Omella, Sabater \& Sabater, 2005) y las relaciones biológicas como la depredación (Binckley \& Resetarits, 2005). El centro de México se caracteriza por la prevalencia de condiciones áridas y semiáridas, en donde 
las redes de drenaje se encuentran integradas por corrientes con flujo de agua permanente y corrientes intermitentes que presentan una dinámica de desecación anual en época de sequía. En estos sistemas la dispersión aérea se ha señalado como uno de los factores más importantes en determinar el establecimiento de organismos en comunidades (Larned et al., 2010), en donde Coleoptera y los insectos hemimetábolos conforman la mayoría de órdenes de insectos acuáticos que presentan una fase adulta aérea con esta capacidad dispersiva.

La mayoría de los estudios sobre las comunidades de insectos acuáticos en el país se enfocan en el estudio del deterioro de los sistemas ribereños usándolos como indicadores biológicos (Pérez-Munguía \& Pineda-López, 2005; Alonso-Eguia Lis, 2007). Mientras que temas relacionados a la ecología, la importancia de los patrones de distribución dentro y entre los cauces, las variables que afectan el establecimiento de especies, así como la escala a la que estas variables tienen efecto, permanecen poco documentadas (Campbell, Arce-Pérez \& Gómez-Anaya, 2008).

Las variables que influyen sobre las comunidades acuáticas tienen efecto en diferentes escalas o filtros espaciales (sensu Poff, 1997). Estos filtros varían desde el nivel de cuenca hasta el de microhábitat e influyen sobre las comunidades de macroinvertebrados acuáticos en sistemas dulceacuícolas (Stendera \& Johnson, 2005; Ligeiro, Melo \& Callisto, 2009; Cardoso de Ávila, Stenert \& Maltchik, 2011). La cuenca es una escala natural bien delimitada, con procesos y características propias: geomorfológicas, hidrológicas, climáticas y de uso de suelo, entre otras. Dentro de esta escala, existen factores bióticos y abióticos que influyen en la presencia y abundancia de los organismos, definiendo la comunidad acuática local (Poff, 1997). Mientras que las diferencias geomorfológicas, de hidroperiodo y de condiciones locales de los cauces, pueden restringir o favorecer la dispersión y establecimiento de organismos (Poff, 1997; Costa \& SánchezMelo, 2008; García-Roger et al., 2011). Finalmente, el nivel de microhábitat contiene una gama de parámetros fisicoquímicos, sustratos y recursos alimentarios que son cruciales para el desarrollo de vida de las comunidades de macroinvertebrados acuáticos y que definen en gran medida su establecimiento (García-Roger et al., 2011).

El objetivo principal de este estudio fue determinar los factores ambientales y la escala a la que éstos afectan la diversidad alfa $(\alpha)$ y beta $(\beta)$ de los órdenes estudiados en ríos permanentes e intermitentes dentro de la cuenca del Río Xichú. La hipótesis formulada fue que las variables ambientales que actúan a nivel de microhábitat dentro de los cauces son las principales determinantes de la diversidad.

\section{MATERIALES Y MÉTODOS}

El área de estudio se encuentra dentro de la región fisiográfica de la Sierra Madre Oriental, en la subprovincia de la Sierra Gorda, dentro de la Reserva de la Biosfera "Sierra Gorda de Guanajuato" por lo que los cauces bajo estudio presentan poco impacto antropogénico. El área corresponde a la cuenca de Xichú (Fig. 1), ubicada al noreste del estado de Guanajuato, en el centro de México. La zona se caracteriza por la prevalencia de condiciones semiáridas de climas secos y semi-secos, con una precipitación promedio anual estimada en $474 \mathrm{~mm}$ (Iracheta, 2009), distribuida entre los meses de mayo y septiembre lo que promueve la existencia de cauces intermitentes. En este sistema se establecieron ocho sitios de muestreo en los principales cauces, cuatro sitios permanentes (Paso de Guillermo, Pablos, Llano Grande y Joya Fría) y cuatro intermitentes (Xichú, Organitos, Llanetes y Huamúchil) que presentan agua de junio a diciembre (Cuadro 1).

Se efectuaron dos muestreos, el primero en el mes de diciembre del 2010, cuando aún todos los cauces presentaban agua. El segundo se realizó a finales de la temporada seca en el mes de junio del 2011, donde solamente los sitios permanentes tenían agua (el número 2 después de las siglas de identificación de los ríos permanentes indica que se refieren al segundo muestreo). En cada sitio se tomaron 


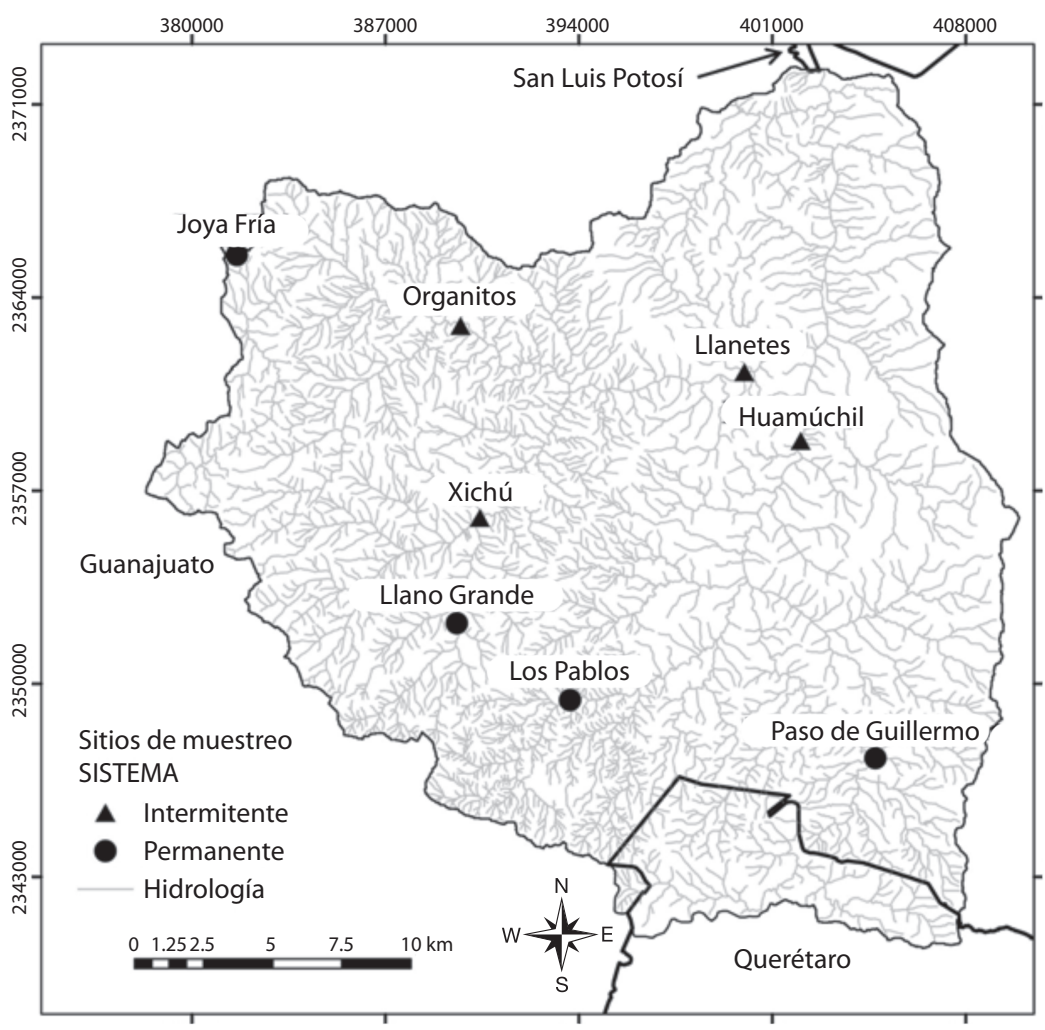

Fig. 1. Ubicación de la Cuenca de Xichú y los sitios de muestreo, Guanajuato, México.

Fig. 1. Location of the Xichú's watershed and sampling sites.

\section{CUADRO 1}

Caracterización de los sitios de estudio en la cuenca Xichú, México.

TABLE 1

Information of study sites from Xichu's Basin, Mexico.

\begin{tabular}{|c|c|c|c|c|c|c|}
\hline Sitio & ID & $\begin{array}{l}\text { Altitud } \\
\text { (msnm) }\end{array}$ & Orden & Tipo de suelo & Uso de suelo circundante & $\begin{array}{l}\text { Tipo de } \\
\text { sistema }\end{array}$ \\
\hline Paso de Guillermo & PG & 1331 & 3 & Volcánico & Agrícola & Permanente \\
\hline Los Pablos & $\mathrm{Pa}$ & 1525 & 4 & Volcánico & $\begin{array}{l}\text { Matorral submontano y pequeños } \\
\text { parches de agricultura }\end{array}$ & Permanente \\
\hline Llano Grande & LG & 1648 & 3 & Volcánico & Bosque galería y agricultura & Permanente \\
\hline Joya Fría & $\mathrm{JF}$ & 2440 & 1 & Volcánico & Bosque pino-encino y agrícola temporal & Permanente \\
\hline Xichú & $\mathrm{Xi}$ & 1286 & 5 & Sedimentario & Asentamientos humanos & Intermitente \\
\hline Organitos & Or & 1250 & 5 & Sedimentario & $\begin{array}{l}\text { Selva baja caducifolia y Agricultura } \\
\text { temporal }\end{array}$ & Intermitente \\
\hline Llanetes & Lla & 926 & 6 & Sedimentario & $\begin{array}{l}\text { Selva baja caducifolia y Agricultura } \\
\text { temporal }\end{array}$ & Intermitente \\
\hline Huamúchil & $\mathrm{Hu}$ & 924 & 5 & Sedimentario & $\begin{array}{l}\text { Selva baja caducifolia y Agricultura } \\
\text { temporal }\end{array}$ & Intermitente \\
\hline
\end{tabular}

ID es un identificador que se usara en adelante para referirse a los sitios. 
ocho muestras en microhábitats diferentes con ayuda de una red tipo "D" con luz de malla de $300 \mu \mathrm{m}$ y un total aproximado de $1.2 \mathrm{~m}^{2}$ de área muestreada. En cada muestra se describió el hábitat con base en el mesohábitat (rápido/ estanque), profundidad del agua (clasificada como muy profundo $>0.75 \mathrm{~m}$. profundo $0.74 \mathrm{~m}$ a 0.50 , somero 0.49 a $0.25 \mathrm{~m}$ y muy somero $<0.24 \mathrm{~m}$ ) y la velocidad de la corriente aparente (clasificada como alta, media, baja y nula). Además, se midió el oxígeno disuelto $(\mathrm{mg} / \mathrm{L})$, temperatura $\left({ }^{\circ} \mathrm{C}\right), \mathrm{pH}$ (unidades de $\mathrm{pH}$ ), salinidad (PSS), turbidez (NTU), potencial óxidoreducción $(\mathrm{mV})$, conductividad específica $(\mathrm{mS} /$ $\mathrm{cm})$ y sólidos disueltos totales $(\mathrm{g} / \mathrm{L})$ con un multiparamétrico marca Quanta ${ }^{\circledR}$.

Las muestras fueron conservadas en campo usando alcohol al 80\%. Los organismos fueron separados en el laboratorio con ayuda de un microscopio estereoscópico y fijados usando alcohol al 70\%. La identificación a nivel de género se basó en Merrit, Cummins \& Berg (2008) para el nivel de familia y género, exceptuando algunos adultos de Coleoptera para los cuales se usaron Arnett \& Thomas (2001), Arnett, Thomas, Skelley \& Frank (2002), Navarrete-Heredia \& Zaragoza-Caballero (2006), así como Arce-Pérez, Novelo-Gutiérrez \& Gómez-Anaya (2002) y Arce-Pérez, GómezAnaya \& Novelo-Gutiérrez (2010). Todos los individuos permanecen en el laboratorio de Monitoreo de la Biodiversidad de la Universidad Autónoma de Querétaro.

Los valores de diversidad alfa, definida como la diversidad que se encuentra a escala local en un sitio de muestreo definido, de extensión pequeña y relativamente homogéneo (Moreno \& Rodríguez, 2010), fueron obtenidos a través de medidas de diversidad verdadera ${ }^{\mathrm{q}} \mathrm{D}$ (Jost, 2007), en donde se define un exponente (q) que determinará la sensibilidad del índice a las abundancias relativas de las especies (Moreno, Barragan, Pineda \& Pavón, 2011). La diversidad de orden $0(\mathrm{q}=0)$, es totalmente independiente de la abundancia y corresponde a la riqueza de especies, mientras que la diversidad de orden 1 ( $\mathrm{q}=1$ ) incluye a las especies en el análisis con un peso proporcional a su abundancia y puede ser expresado como el exponencial de H' (índice de Shannon). Uno de los componentes más importantes a entender dentro de las comunidades es la diversidad $\beta$, que en este trabajo se define como el cambio en la composición taxonómica entre sitios de muestreo, y se estimó mediante un análisis de similitud, cuyos valores se representan en un dendrograma, donde las muestras son agrupadas con base en la similitud de sus composiciones. La interpretación de los grupos conformados en el dendrograma fue subjetiva y la decisión sobre cuáles grupos reportar está basada en un corte arbitrario dictado por la disimilitud. Estos análisis de agrupamiento se realizaron con el paquete estadístico PAST (Hammer, Harper \& Ryan, 2001), usando la medida de distancia de Jaccard $\left(I_{J}\right)$ con el algoritmo UPGMA (Unweighted Pair Group Method using Arithmetic averages).

La partición aditiva de la diversidad fue usada para descomponer la variación total de la composición de la comunidad (diversidad $\gamma$ o regional) en sus componentes alfa y beta evaluando cuatro escalas o niveles para determinar cuál de ellas tiene mayor influencia sobre la comunidad. El primer nivel corresponde al microhábitat, que en este caso se refiere a cada muestra tomada, y que dentro del modelo corresponde a $\alpha 1$ y $\beta 1$. El siguiente nivel es el mesohábitat ( $\beta 2$ ) representado por los rápidos y estanques de cada sitio. El tercer nivel corresponde a cada sitio de muestreo ( $\beta 3$ ) y el nivel más alto corresponde al tipo de sistema ( $\beta 4)$, permanente o intermitente (temporada para el análisis de sitios permanentes entre temporadas). Este análisis permite evaluar el peso relativo de cada nivel con base en el valor de $\alpha$ y $\beta$ (sensu Crist, Veech, Gering \& Summerville, 2003), así como determinar si la diversidad de especies es producto del azar, o si puede ser atribuida a factores propios del paisaje o de la naturaleza de los taxones. Los valores observados son comparados con un modelo nulo, el cual es generado a partir de 1000 interacciones aleatorias (Crist et al., 2003; Cardoso de Ávila, Stenert \& Maltchik, 2011). Para este análisis 
se utilizó el programa Partition v3.0 (Veech \& Crist, 2009).

Las variables fisicoquímicas fueron analizadas mediante un análisis de componentes principales (ACP), usando el programa estadístico JMP 8.0, para determinar relaciones entre variables y eliminar aquellas interrelacionadas. Se practicó un Análisis de Correspondencia Canónica (ACC) que está diseñado para extraer gradientes de conjuntos de datos ambientales graficados en un diagrama de ordenación, donde los gradientes son la base para describir y visualizar las diferentes preferencias de hábitat de los taxones (ter Braak \& Verdonschot, 1995). El ACC se realizó con el programa PAST (Hammer et al., 2001), con variables de 4 tipos: 1) fisicoquímicas; 2) ambientales a nivel de hábitat: mesohábitat (rápidos o estanques), profundidad del agua y velocidad de corriente; 3) ambientales a nivel de ríos: orden del cauce, altitud, pendiente, uso de suelo circundante y calidad ecológica. Estas últimas obtenidas del Programa de Manejo y Conservación de la Reserva de la Biósfera de la Sierra Gorda de Guanajuato, por medio de sistemas de información geográfica. Finalmente, 4) tipo de sistema, variable considerada a nivel de paisaje, que se refiere al flujo de agua en los cauces (permanente o intermitente). El análisis se realizó con un total de 15 variables: oxígeno disuelto, temperatura, $\mathrm{pH}$, turbidez, potencial óxidoreducción, conductividad específica, profundidad, velocidad de la corriente, mesohábitat, hidroperiodicidad, pendiente, orden del cauce, uso de suelo circundante, altitud y calidad ecológica. Esta última variable se tomó de un modelo de sistema de información geográfica y está basada en criterios de fragilidad natural, calidad del agua, erosión y antropización de la cobertura vegetal. Se define como una condición que se refiere al mantenimiento de los elementos y procesos geoecológicos dentro de un ecosistema o unidad natural determinada, como la cuenca. De esta manera, cuando se introduce un agente de presión que deteriora cierto recurso, se modifica la estructura de los elementos y ciertos procesos se ven modificados, lo que reduce la calidad de la unidad natural (UAQ, 2012).

\section{RESULTADOS}

Se cuantificaron un total de 34746 individuos, dentro de 35 familias y 89 géneros de los grupos estudiados, en donde se encontraron presentes otros ordenes no cuantificados para este estudio como: Diptera y Trichoptera. El orden Coleoptera se presentó como el más rico con 46 géneros, seguido de Odonata con 18, Hemiptera y Ephemeroptera presentaron 12 géneros cada uno y por último Plecoptera con un género. Cuatro especímenes de los taxa Grapocorixini (Hemiptera), Lampyridae, Ptiliidae y Sphaeriniinae (Coleoptera) no pudieron ser identificados a nivel de género debido a la falta de claves para su determinación, pero se incluyeron en los análisis como morfogéneros. La distribución de las abundancias mostró una alta disparidad con el $29 \%$ de los géneros exhibiendo bajas abundancias, presentándose como individuos únicos o dobles. Mientras que el género Baetis (Ephemeroptera) fue dominante concentrando el $40 \%$ de la abundancia.

La mayor riqueza genérica se presentó en los eventos de muestreo $\mathrm{Hu}$ y $\mathrm{Pa}-2$ con 43 géneros cada uno, mientras que el valor más bajo de riqueza se encontró en Xi con 20 géneros. Los valores de diversidad verdadera de nivel 1, nos indican que las diversidades más altas se presentaron generalmente en los sitios permanentes durante el segundo muestreo, en donde el sitio más diverso presenta una diversidad 3.11 veces mayor que el sitio menos diverso (Cuadro 2). La magnitud en las diferencias de diversidad para los sitios intermitentes del primer muestreo se presentó del orden de 2.58, en donde el sitio Lla se presenta como el más diverso con un valor de $9.87 \mathrm{y}$ el sitio menos diverso $\mathrm{Xi}$, con 3.8 (Cuadro 2).

Los análisis de similitud mostraron una marcada diferencia de JF con los demás sitios, presentando un valor inferior a $I_{J}=0.25$ en ambos dendrogramas (Fig. 2A). Además el análisis entre temporadas agrupó ambos muestreos en JF, separándose de los otros sitios 
CUADRO 2

Valores de abundancia y diversidad obtenidos con medidas de diversidad verdadera, para los sitios de estudio en la cuenca.

TABLE 2

Abundance and diversity values obtained from true diversity measures.

\begin{tabular}{|c|c|c|c|c|c|c|c|c|c|c|c|c|}
\hline & \multicolumn{8}{|c|}{ Sitios Permanentes } & \multirow{2}{*}{\multicolumn{4}{|c|}{$\begin{array}{c}\text { Sitios Intermitentes } \\
1^{\text {er }} \text { Muestreo }\end{array}$}} \\
\hline & \multicolumn{4}{|c|}{$1^{\mathrm{er}}$ Muestreo } & \multicolumn{4}{|c|}{$2^{\text {do }}$ Muestreo } & & & & \\
\hline & PG & $\mathrm{Pa}$ & LG & JF & PG-2 & $\mathrm{Pa}-2$ & LG-2 & JF-2 & $\mathrm{Hu}$ & $\mathrm{Xi}$ & Or & Lla \\
\hline $\mathrm{n}$ & 5583 & 3676 & 2814 & 884 & 932 & 2595 & 1616 & 2334 & 4704 & 604 & 8324 & 679 \\
\hline${ }^{0} D$ & 32 & 29 & 38 & 27 & 35 & 43 & 30 & 24 & 43 & 20 & 27 & 28 \\
\hline${ }^{1} D$ & 4.85 & 6.58 & 8.67 & 9.58 & 9.99 & 10.63 & 6.91 & 3.41 & 5.65 & 3.78 & 3.80 & 9.87 \\
\hline$\gamma$ & & & & & & 89 & & & & & & \\
\hline
\end{tabular}

Abundancia (n), diversidad de orden " 0 " o riqueza $\left({ }^{0} D\right)$, diversidad de orden $1\left({ }^{1} D\right)$ y diversidad regional $(\gamma)$.Abundance (n), order " 0 " diversity or richness $\left({ }^{0} D\right)$, order 1 diversity $\left({ }^{1} D\right)$ and regional diversity $(\gamma)$.
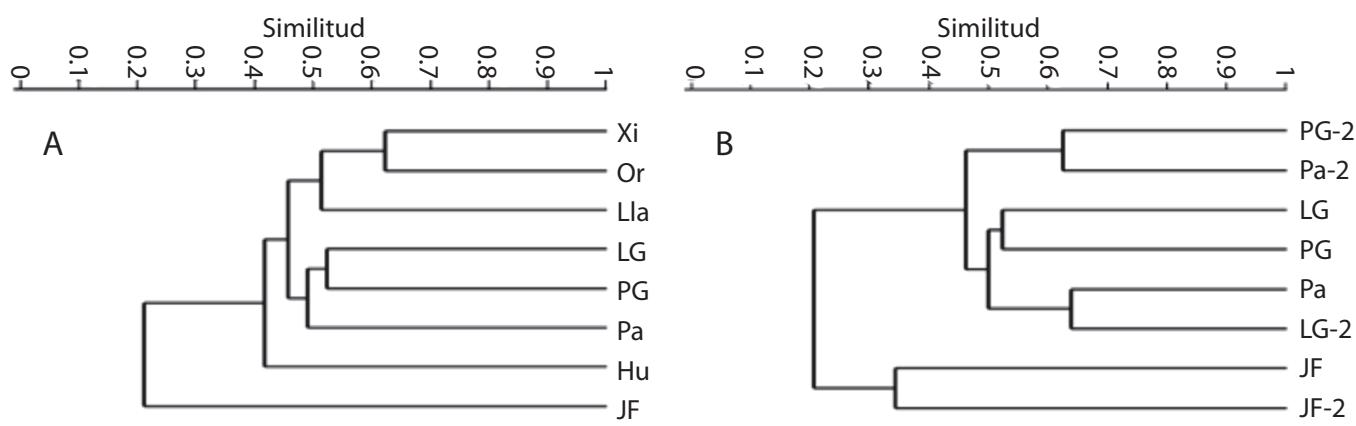

Fig. 2. Análisis de agrupamiento realizado con el coeficiente de similitud de Jaccard entre sitios de diferentes tipos de sistemas (a) y sitios permanentes muestreados en distinta temporada (b).

Fig. 2. Cluster analysis based on Jaccard similarity coefficient for sites from different systems (a) and permanent sites between seasons $(b)$.

permanentes (Fig. 2B). En los agrupamientos observados entre sitios de diferentes tipos de sistemas (Fig. 2A), se encontró una clara diferenciación entre los sitios permanentes (LG, PG y Pa) e intermitentes (Xi, Or y Lla), indicando una similitud en la composición de sus comunidades con valor de $I_{J}=0.46$. Entre los ríos intermitentes, el sitio $\mathrm{Hu}$ fue el único separado de su grupo, presentándose como el segundo sitio más disímil con $I_{J}=0.41$. Para los sitios permanentes entre muestreos (Fig. 2B), la diferenciación entre temporadas no es clara. El sitio LG-2 fue agrupado con los sitios del primer muestreo, presentando incluso el mayor valor de similitud $\left(I_{J}=0.64\right)$ con el sitio Pa del primer muestreo, mientras que los sitios PG-2 y Pa-2 se encuentran formando una agrupación aparte, presentando un valor $I_{J}=0.62$.

Los niveles del análisis multiescalar de la partición aditiva de la diversidad, mostraron que los sitios, sistemas y temporadas aportan la mayor cantidad de diversidad, lo que refleja una alta agregación de insectos en las escalas finas y marcadas diferencias en la composición de insectos entre sitios, tipo de sistema y temporada (Fig. 3). En el caso del análisis de tipo de sistema (Fig. 3A), los niveles de $\alpha 1$ y $\beta 1$ fueron menores a los esperados ( $p>0.999)$, con el $29.65 \%$ de la diversidad global, mientras que $\beta 3$ y $\beta 4$ se presentaron mayores a los esperados 
A

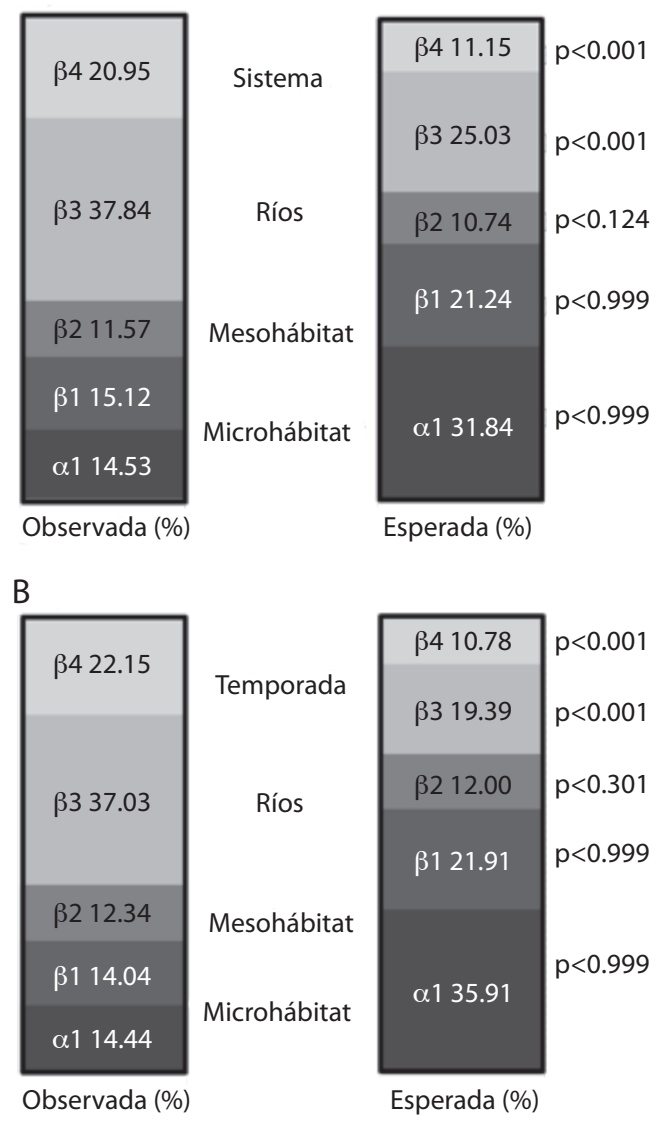

Fig. 3. Diversidad multiescalar observada y esperada, expresadas en porcentaje del total de la diversidad global. a) $\gamma=74$ géneros y b) $=79$ géneros.

Fig. 3. Observed and expected multiscaled diversity expressed as percentages values of global diversity. a) $\gamma=74$ genera and b) $=79$ genera.

( $\mathrm{p}<0.001)$ y con la mayor diversidad $58.79 \%$, mientras que $\beta 2$ no es diferente al esperado $(\mathrm{p}<0.124)$. La partición de la diversidad entre temporadas y sitios (Fig. 3B) aportó 59.18\% de la diversidad global y son mayores a los esperados $(\mathrm{p}<0.001)$, mientras que los niveles finos $\alpha 1$ y $\beta 1$ reunieron el $28.48 \%$ con menor proporción a lo esperado ( $p>0.999)$. La diversidad observada del nivel $\beta 2$ para la temporada no presentó diferencia significativa con los valores esperados $(\mathrm{p}<0.301)$.
Los primeros tres componentes del ACP mostraron una varianza explicada del $79 \%$, (Eje 1: Eigenvalor $=3.65, \%=45.58$; eje 2: Eigenvalor $=1.72, \%=21.52$; eje 3 : Eigenvalor $=0.96, \%=12.06$ ), en donde las variables más fuertemente asociadas al primer componente fueron: conductividad (0.51), salinidad (0.51) y solidos disueltos totales (0.5); en el segundo se encuentran las variables de temperatura $(0.45), \mathrm{pH}(-0.42)$ y potencial oxidoreducción (0.64), así como la turbidez (0.95) para el tercer componente. Para el ACC fueron descartadas las variables de salinidad y solidos disueltos totales debido a su alta interrelación, manteniéndose la variable de conductividad ya que representa el conjunto de solidos que se encuentran en la columna de agua.

El ACC reportó el $57.2 \%$ de la varianza explicada en los tres primeros ejes, a través de los valores de la inercia de la matriz. Las variables con mayor peso explicativo fueron mesohábitat $(-0.656)$ y velocidad $(-0.771)$ en el primer eje; la altura (0.586), la calidad ecológica (0.46), el oxígeno disuelto $(-0.463)$ y el orden del cauce (-0.486) para el segundo y el potencial óxido-reducción (0.332) para el tercero. En el diagrama de ordenación (Fig. 4), se observó una tendencia de las muestras de JF a separarse en la parte superior, este sitio es el de mayor altura, mejor calidad ecológica y presenta la mayor rareza con 12 taxa únicos al sitio (Helocombus, Helophorus, Laccobius, Stenus, Zonagrion, Dytiscus, Neoporus, Rhionaeschna multicolor, Ptiliidae, Sanfilippodytes, Acuarius y Suniocharis). Los eventos del segundo muestreo presentaron una cantidad mayor de estanques y de velocidad baja o nula, dispersándose hacia el lado derecho de la ordenación. No se observó un patrón definido en relación a los demás géneros.

\section{DISCUSIÓN}

El centro de México se ha reconocido por albergar una gran diversidad biológica producto de su compleja historia ecológica (Halffter, Llorente-Bousquets \& Morrone, 2008). Sin embargo, en términos de su entomofauna, la 


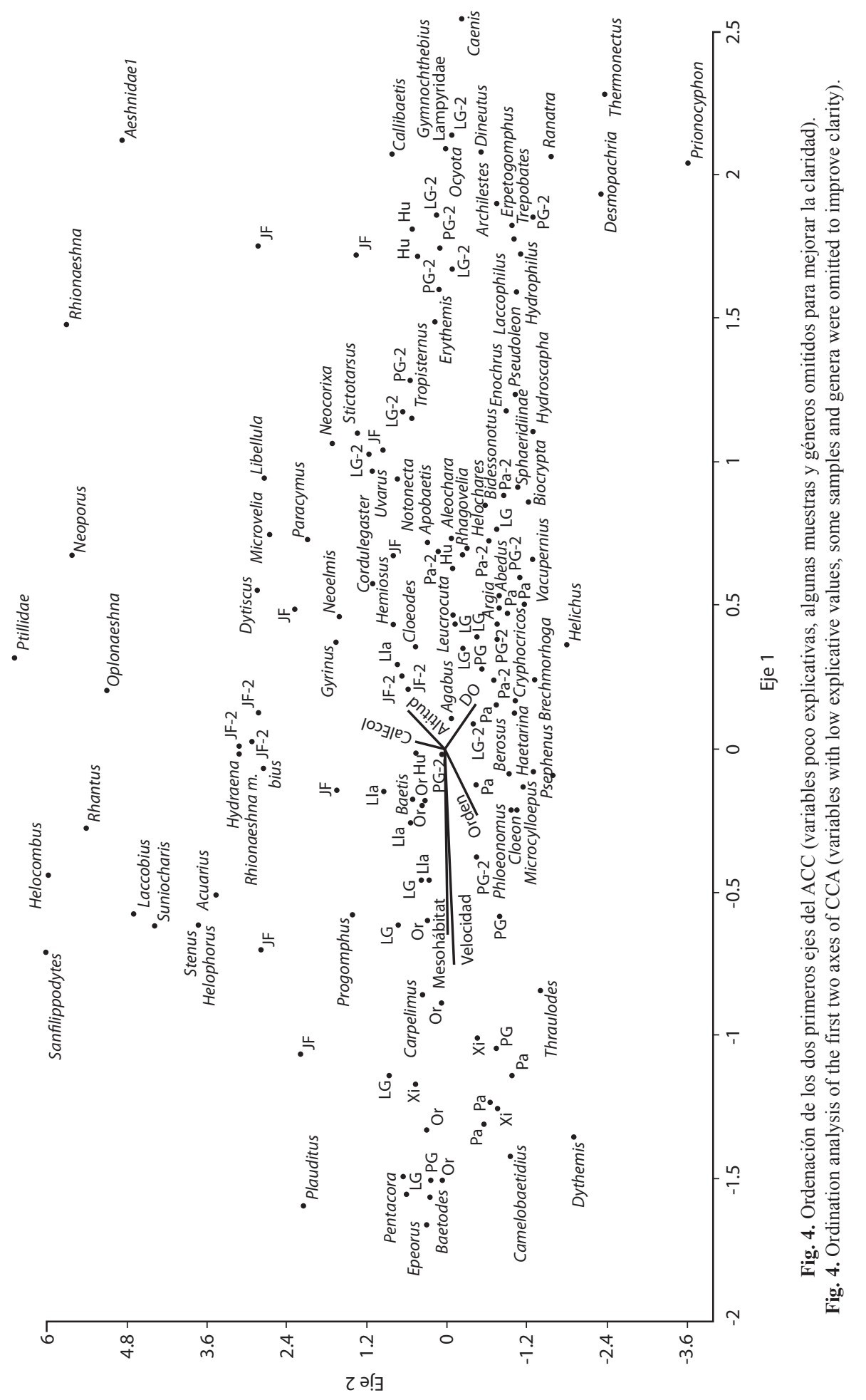


falta de exploración en la zona ha ocasionado que los listados de insectos se encuentren aún sub-representados. El presente estudio denota una alta riqueza de géneros de insectos acuáticos, comparada con la reportada en otros trabajos (Arce-Pérez \& Novelo, 1991; Arce-Pérez et al., 2002, 2010), aun teniendo la cuenca bajo estudio un área menor (Juárez-Flores, 2007; Dinger et al., 2005). La época seca, se caracterizó por una mayor riqueza de géneros, lo que se podría explicar por la ausencia de las inundaciones que se presentan en la temporada de lluvias, que actúan como una alteración provocando mortalidad e inestabilidad en la comunidad (Townsend, 1989).

De acuerdo con los análisis de similitud, las comunidades de insectos establecidos en sitios permanentes, presentan grados de similitud con los sitios intermitentes por debajo $I_{J}<0.5$, presentando diferencias importantes en la composición taxonómica, que se deben principalmente a la presencia de una mayor diversidad de géneros de los órdenes estudiados en sitios permanentes. Esto sugiere la existencia de variaciones en los rasgos biológicos de los insectos que los facultan para colonizar y establecerse con éxito en sitios con diferente hidroperiodo (Campbell et al., 2008). De la misma manera, se presenta variación en las comunidades de sitios permanentes entre temporadas (Fig. 2B), lo que puede indicar una diferenciación en la época de emergencia del medio acuático de los taxones. Sin embargo, estudios adicionales son necesarios para aceptar o descartar este argumento.

La partición escalar de la diversidad $\gamma$, en $\alpha$ y $\beta$ indica que el componente $\beta$ aportó la mayor diversidad, principalmente en las escalas $\beta 3$ y $\beta 4$, que corresponden con ríos, tipo de sistema y temporada. De la misma manera, Cardoso de Ávila, Stenert \& Maltchik (2011) y Stendera \& Johnson (2005), encontraron que las escalas o filtros espaciales mayores son los que definen el cambio en la composición de macroinvertebrados acuáticos en sistemas dulceacuícolas y no los filtros relacionados con las escalas más finas (Ligeiro et al., 2009). En la cuenca del Río Xichú existe una alta diversidad $\beta$ espacial y temporal de coleópteros e insectos hemimetábolos, debida a los bajos valores de similitud entre sitios de muestreo $\left(I_{J}<0.65\right)$ y altos valores de diversidad $\beta$ en la partición multiescalar. Por ello, el componente $\beta$ es muy importante a nivel de paisaje en México (Novelo-Gutiérrez \& Gómez-Anaya, 2009).

Las variables explicativas del ACC indican que la distribución y establecimiento de los organismos no está determinada por un solo tipo de variables, si no a la interacción de variables que se encuentran actuando a distintas escalas: tipo de sistema/temporada, cauces, mesohábitat y microhábitat (Pires, Cowx \& Coehlo, 2000). La diferencia entre la cantidad de rápidos $\mathrm{y}$ estanques a lo largo del año se presenta como un factor importante para el desarrollo y establecimiento de los insectos acuáticos en la cuenca. La disminución en la diversidad de hábitats de rápidos debido al cese del flujo, ocasiona que exista una mayor cantidad de organismos con adaptaciones necesarias para sobrevivir en ríos intermitentes y de bajo flujo (Bonada, 2008), como la capacidad de trasladarse grandes distancias, ciclos de vida cortos (bivoltinos o multivoltinos) y respiración aérea (Campbell et al., 2008). La diversidad $\beta$ encontrada a nivel de sitios de muestreo, resulta de diferencias ambientales entre ríos y arroyos, debido a que el orden del cauce y la altitud se relacionan con otras variables, como la temperatura, tipo de vegetación, ancho de la zona ribereña, pendiente del cauce. Estas variables funcionan como filtros que actúan de manera diferenciada entre los organismos, dependiendo de los rasgos biológicos que presentan (Poff, 1997) y promoviendo un establecimiento diversificado entre los cauces.

La diversidad $\beta$ es el principal componente de la diversidad de la cuenca del Río Xichú, en donde las escalas de cauce y paisaje determinan de manera mayoritaria la diversidad. Esto se debe a las diferencias ambientales que se presentan entre los cauces y a las diferencias en requerimientos ecológicos de los organismos. Los resultados presentados indican que es a éstos niveles donde deben enfocarse los planes de conservación y manejo de los 
sistemas dulceacuícolas. A nivel de paisaje, los resultados del índice de similitud y de la riqueza de los sitios, sugieren que los sitios permanentes se funcionan como fuente de especies para los sitios intermitentes. Esto sugiere la necesidad de nuevas estrategias de conservación basadas en la diversidad $\beta$, que permitan la conexión entre áreas, con la finalidad de asegurar la viabilidad de las comunidades en sitios intermitentes.

\section{AGRADECIMIENTOS}

Los autores agradecen al Consejo Nacional de Ciencia y Tecnología por el financiamiento otorgado. A Rodolfo Novelo Gutiérrez por su desinteresada ayuda en la determinación de especímenes de Odonata, a Alonso Ramírez por todo su apoyo, orientación y mejoras al documento. Finalmente agradecemos a los revisores por sus valiosos aportes y comentarios que mejoraron sustancialmente este trabajo.

\section{RESUMEN}

Los sistemas lóticos integrados por cauces temporales representan una alta proporción de las aguas continentales del planeta, no obstante se encuentran entre los sistemas menos estudiados a nivel mundial. En cuanto a diversidad entomológica, el centro de México -donde se ubican estos sistemas- permanece poco estudiado a pesar del alto potencial que presenta la zona de albergar una alta diversidad y de los vínculos importantes que este grupo desempeña en los flujos de energía de los ecosistemas acuáticos. El objetivo del presente trabajo fue analizar las variables ambientales que determinan los cambios en la diversidad y las escalas en que tienen efecto, en las comunidades de insectos acuáticos de un sistema fluvial semiárido del centro de México. Se registraron un total de 89 géneros, con el orden Coleoptera presentando la mayor riqueza. Los valores más altos de diversidad alfa se encontraron sobre sitios permanentes en la temporada de sequía ( $\left.{ }^{1} \mathrm{D}: 10.63\right)$, lo que se atribuye a la mayor estabilidad que presentan los sistemas fluviales en esta temporada. Las comunidades establecidas sobre cauces permanentes e intermitentes presentaron baja similitud $\left(\mathrm{I}_{\mathrm{j}}<0.45\right)$, indicando un alto recambio entre comunidades establecidas sobre cauces con diferente hidroperiodo. Los análisis de distribución ("partición") de la diversidad indican que el mayor aporte a la diversidad regional se da por cambios en la composición de géneros a nivel de ríos (37\%), entre temporadas (22\%) y por el tipo de sistema (21\%). De acuerdo con el Análisis de
Correlación Canónica las variables de mesohábitat, velocidad de corriente, calidad ecológica y orden del cauce son las que mejor explican la variación en la presencia de los taxones. Los resultados sugieren que los sitios permanentes se encuentran actuando como fuente de especies de los sitios intermitentes y que los esfuerzos de conservación en la región deben implementarse a escala de paisaje.

Palabras clave: división de la diversidad, diversidad beta, hidroperiodo, cauces temporales, escalas ecológicas.

\section{REFERENCIAS}

Acuña, V., Muñoz, I., Giorgi, A., Omella, M., Sabater, F., \& Sabater, S. (2005). Drought and postdrought recovery cycles in an intermittent Mediterranean stream: structural and functional aspects. Journal of the North America Benthological Society, 24, 919-933.

Alonso-EguíaLis, P. E. (2007). Importancia del estudio de la entomofauna acuática para la conservación y el manejo sustentable de sistemas dulceacuícolas de México. In R. Novelo-Gutiérrez \& P. E. AlonsoEguíaLis (Eds.). Simposio Internacional de Entomología Acuática: estado actual de conocimiento y aplicación. Morelos: Instituto Mexicano de Tecnología del Agua, Sociedad Mexicana de Entomología.

Arce-Pérez, R., Gómez-Anaya, J. A., \& Novelo-Gutiérrez, R. (2010). Coleópteros acuáticos de la zona de influencia de la central hidroeléctrica "Ing. Fernando Hiriart Balderrama" (C.H. Zimapan), Hidalgo, México. II. Coleoptera: Polyphaga y Myxophaga. Acta Zoológica Mexicana, 26, 639-667.

Arce-Pérez, R., Novelo-Gutiérrez, R., \& Gómez-Anaya, J. A. (2002). Coleópteros acuáticos de la zona de influencia de la Central Hidroeléctrica "Ing. Fernando Hiriart Balderrama” (PH. Zimapán), Hidalgo, México I. (Coleoptera: Adephaga: Dytiscidae, Haliplidae, Gyrinidae). Folia Entomológica Mexicana, 41, 229-248.

Arce Pérez, R., \& Novelo Gutiérrez, R. (1991). Coleópteros Acuáticos de la reserva de la biosfera de la Michilia, Durango, México. Folia Entomología Mexicana, 81, 341-344.

Arnett, R. H., \& Thomas, M. C. (2001). American Beetles. Archostemata, Myxophaga, Adephaga, Polyphaga: Staphyliniformia. Vol. 1. CRC. EUA.

Arnett, R. H., Thomas, M. C., Skelley, P. E., \& Frank, J. H. (2002). American Beetles. Polyphaga: Scarabaeoidea trough Curculionidea. Vol. 2. CRC. EUA.

Binckley, C. A., \& Resetarits, W. J. (2005). Habitat selection determines abundance, richness and species composition of beetles in aquatic communities. Biology Letters, 1, 370-374. 
Bonada, N., Rieradevall, M., \& Prat, N. (2007). Macroinvertebrate community and biological traits related to flow permanence in a Mediterranean river network. Hydrobiologia, 589, 91-106.

Bonada, N., Rieradevall, M., Dallas, H., Davis, J., Day, J., Figueroa, R., Resh, V. H., \& Prat, N. (2008). Multiscale assessment of macroinvertebrate richness and composition in Mediterranean-climate rivers. Freshwater Biology, 53, 772-788.

ter Braak, C. J. E., \& Verdonschot, P. E. M. (1995). Canonical correspondence analysis and related multivariate methods in aquatic ecology. Aquatic Sciences, 57, 255-289.

Campbell, W. B., Arce-Pérez, R., \& Gómez-Anaya, J. A. (2008). Taxonomic distinctness and aquatic Coleoptera: comparing a perennial and intermittent stream with differing geomorphologies in Hidalgo, México. Aquatic Ecology, 42, 103-113.

Cardoso de Ávila, A., Stenert, C., \& Maltchik, L. (2011). Partitioning macroinvertebrate diversity across different spatial scales in southern Brazil coastal wetlands. Wetlands, 31, 459-469.

Costa, S. S., \& Sanches-Melo, A. (2008). Beta diversity in stream macroinvertebrate assemblages: among-site and among-microhabitat components. Hydrobiologia, 598, 131-138.

Crist, T. O., Veech, J. A., Gering, J. C., \& Summerville, K. S. (2003). Partitioning species diversity across landscapes and regions: a hierarchical analysis of $\alpha, \beta$, and $\gamma$ diversity. The American Naturalist, 162, 734-743.

Dinger, E., Cohen, A., Hendrickson, D., \& Marks, J. (2005). Aquatic invertebrates of Cuatro Cienegas, Coahuila, México: Natives and exotics". The Southwestern Naturalist, 50, 237-246.

Fairchild, G. W., Cruz, J., \& Faulds, A. M. (2003). Microhabitat and landscape influences on aquatic beetle assemblages in a cluster of temporary and permanent ponds. Journal of the North American Benthological Society, 22, 224-240.

Feld, A. K., \& Hering, D. (2007). Community structure or function: effects of environmental stress on benthic macroinvertebrates at different spatial scales. Freshwater Biology, 52, 1380-1399.

García-Roger, E., Sánchez-Montoya, M., Gómez, R., Suárez, M. L., Vidal-Abarca, M. R., Latron, J., Rieradevall, M., \& Prat, N. (2011). Do seasonal changes in habitat features influence aquatic macroinvertebrate assemblages in perennial versus temporary Mediterranean streams? Aquatic Science, 73, 567-579.

Halffter, G., Llorente-Bousquets, J., \& Morrone, J. (2008). La perspectiva biogeográfica histórica. In Capital natural de México, vol. I: Conocimiento actual de la biodiversidad (pp. 67-86). CONABIO: México.
Hammer, O., Harper, D. A. T., \& Ryan, P. D. (2001). PAST: Paleontological Statistics software package for education and data analysis. Paleontología Electrónica 4, 9.

Juárez-Flores, J. (2007). Caracterización de la cuenca del río Metztitlán (Hidalgo, México) con base en la entomofauna acuática y condiciones limnológicas. (Tesis de maestría). Universidad Autónoma Metropolitana, Iztapalapa, México.

Jost, L. (2007). Partitioning diversity into independent alpha and beta components. Ecology, 10, 2427-2439.

Iracheta, C. A. X. (2009). Planes Región 2035 del Estado de Guanajuato. Región I Noroeste. Gobierno del estado de Guanajuato, México.

Klečka, J. (2008). The structure and dynamics of a water beetle community in a semipermanent wetland (Vrbenské rybniky Nature Reserve, South Bohemia). (Tesis de Pregrado). Facultad de Ciencias, Universidad del Sur de Bohemia, České Budějovice, República Checa.

Ligeiro, R., Melo, A. S., \& Callisto, M. (2010). Spatial scale and the diversity of macroinvertebrates in a neotropical catchment. Freshwater Biology, 55, 424-435.

Larned, S., Thibault, D., Arscott, D., \& Tockner, K. (2010). Emerging concepts in temporary-river ecology. Special Review. Freshwater Biology, 55, 717-738.

Llorente-Bousquets, J., Gonzales, E., García, A. N., \& Cordero, C. (1996). Breve panorama de la taxonomía de artrópodos en México. In J. Llorente-Bousquets, A. N. García \& E. Gonzales (Eds.), Biodiversidad, taxonomía y biogeografia de artrópodos de México. Hacia una sintesis de su conocimiento. Volumen I. México: Universidad Autónoma de México.

Merrit, R. W., Cummins, K. W., \& Berg, M. B. (2008). An introduction to the aquatic insect of North America. Fourth edition. EUA: Kendall/Hunt.

Mittermeier, R. A., Farrel, T. A., Harrison, I. J., Upgren, A. J., Brooks, T. M., Contreras-Macbeath, T., Sneider, R., Oberfeld, F., Rosenberg, A. A., Boltz, F., Gascon, C., \& Langrand, O. (2011). Agua dulce la esencia de la vida. China: CEMEX y iLCP.

Moreno, C. E., Barragan, F., Pineda, E., \& Pavón, N. (2011). Reanálisis de diversidad alfa, alternativas para interpretar y comparar información sobre comunidades ecológicas. Revista mexicana de biodiversidad, 82, 1249-1261.

Moreno, C. E., \& Rodríguez, P. (2010). A consistent terminology for quantifying species diversity? Oecologia, 163, 279-282.

Navarrete-Heredia, J. L., Newton, A. F., Thayer, M. K., Ashe, J. S., \& Chandler, D. S. (2002). Guía ilustrada para los géneros de Staphylinidae (Coleoptera) de México. Guadalajara: Universidad de Guadalajara y CONABIO. 
Novelo-Gutiérrez, R., \& Gómez-Anaya, J. A. (2009). A comparative study of Odonata (Insecta) assemblages along an altitudinal gradient in the sierra de Coalcomán Mountains, Michoacán, México. Biodiversity Conservation, 18, 679-698.

Pérez-Munguia, R. M., \& Pineda-López, R. (2005). Diseño de un índice de integridad para rios y arroyos del centro de México, usando las asociaciones de macroinvertebrados. México: Entomología Mexicana.

Pires, A. M., Cowx, I. G., \& Coelho, M. M. (2000). Benthic macroinvertebrate communities of intermittent streams in the middle reaches of the Guadiana Basin (Portugal). Hydrobiología, 435, 167-175.

Poff, N. L. (1997). Landscape filters and species traits: towards mechanistic understanding and prediction in stream ecology. Journal of the North America Benthological Society, 16, 391-409.

Stendera, S. E., \& Johnson, R. K. (2005). Additive partitioning of aquatic invertebrate species diversity across multiple spatial scales. Freshwater Biology, 50, 1360-1375.

Veech, J. A., \& Crist, T. O. (2009). Software for partitioning species diversity. Version 3.0.

Townsend, C. (1989). The patch dynamic concept of stream community ecology. Journal of the North America Bentological Society, 8, 36-50.

UAQ. (2012). Programa de manejo y conservación de la Reserva de la Biósfera de la Sierra Gorda de Guanajuato (Borrador). México: Universidad Autónoma de Querétaro-Comisión Nacional de Áreas Naturales Protegidas. 